\title{
Prone Airway Management in Patients with Pressure Ulcers
}

\section{Linton $\mathrm{P}^{1}$, Kim I ${ }^{1}$, Ayello EA ${ }^{2}$, Miller $\mathrm{S}^{1}$ and $\mathrm{O}^{\prime}$ Neill DK ${ }^{* 1}$}

${ }^{1}$ Department of Anesthesiology, New York University School of Medicine, New York, United States

${ }^{2}$ Excelsior College, School of Nursing, Albany, United States

*Corresponding author: O’Neill DK, Department of Anesthesiology, New York University School of Medicine, New York University Langone Medical Center, 550 First Avenue, TH 534G, New York, NY 10016, United States, Fax: 212-263-7254, Tel: 212-263-5072, E-mail: Daniel.ONeill@nyumc.org

Citation: Linton P, Kim I, Ayello EA, Miller S, O’Neill DK (2016) Prone Airway Management in Patients with Pressure Ulcers. J Anesth Pati Care 1(2): 201. doi: 10.15744/2456-5490.1.201

Received Date: December 16, 2015 Accepted Date: April 22, 2016 Published Date: April 25, 2016

\begin{abstract}
Pressure ulcers are a critical but manageable complication commonly seen in bed-bound patients. Utilizing the Wound Bed Preparation (WBP) model, it is often necessary to perform surgical debridement in prone position for patients with stage III or IV sacral pressure ulcers to avoid infection and further complications. Intraoperative anesthesia care for patients undergoing debridement in prone position presents several challenges due to changes in physiology, higher level of care required to ensure airway protection, and complications associated with anesthetic drugs - most commonly the use of neuromuscular blocker (NMB). We hypothesize that airway management in these patients can safely be accomplished by regional anesthesia with sedation as needed, or if necessary, general endotracheal anesthesia without the use of NMB. With retrospective review of our data, we propose an alternate algorithm in performing prone position endotracheal anesthesia. The major difference in our algorithm is eliminating the use of NMB while promoting the use of video laryngoscopy during intubation. We believe our approach leads to minimizing the amount of anesthetic drugs administered and avoiding its adverse effects, while also minimizing the duration of post-operative mechanical ventilation. This allows the anesthesiologist to provide safe and effective intraoperative care even in severely ill patients requiring surgical intervention. KeyWords: Airway; Prone; Wounds; Pressure ulcers; Anesthesia
\end{abstract}

\section{Introduction}

Pressure ulcers are a common and serious complication with a prevalence ranging from $11.5 \%$ in acute care to rates of $27.7 \%$ and $32.2 \%$ in long-term care settings. In the past decade, the incidence of pressure ulcers has increased by as much as $80 \%$ [1-4]. In one large survey, facility-acquired pressure ulcers in acute care settings has been reported as ranging from 5.0\% to $6.4 \%$, with the National Pressure Ulcer Advisory Panel (NPUAP) reporting a prevalence ranging from $0 \%$ to $15.8 \%$ [1,3]. Pressure ulcers can lead to prolonged hospital stays and an increased risk of secondary complications such as sepsis [5]. It is estimated that 60,000 patients in the U.S. die every year from causes related to hospital-acquired pressure ulcers [6]. The majority of ulcers occur over bony prominences approximately $32 \%$ develop in the sacral region [7].

The Wound Bed Preparation (WBP) paradigm has been well accepted within the wound care community as a model to use for treatment and healing of chronic wounds including pressure ulcers $[8,9]$. When patients present with stage III or IV pressure ulcers, it is often necessary within the WBP component of local wound care to perform surgical debridement as necrotic tissues can serve as a medium for infection and impair wound healing [7]. However, surgical intervention carries higher inherent risks than medical therapy since these patients tend to have more comorbidities than an average healthy patient. A review of patient data revealed that patients with chronic wounds have an average score of 3.09 on the American Society of Anesthesiologists (ASA) physical status classification scale compared with the overall average score of 2.03 for all anesthesia patients at a major university hospital [10].

If surgery is deemed necessary, pressure ulcers in the sacrum can be debrided in prone or lateral position with a wide variety of anesthetic techniques. This presents numerous challenges because of physiologic changes in the prone position as well as the belief that sacral ulcers often require significantly different anesthetic regimens from ulcers in other locations [11,12]. Traditionally, inducing general anesthesia with endotracheal intubation and neuromuscular blocker (NMB) while the patient is supine and repositioning the patient into the prone position has been the gold standard $[13,14]$. However, administering NMB can lead to complications, such as postoperative hypoventilation, re-intubation, aspiration, and tachypnea $[15,16]$. A recent study has suggested that regional anesthesia with sedation as needed may be the most effective regimen for some patients undergoing surgical debridement in prone or lateral position [10]. This is in accordance with studies that have demonstrated that regional anesthesia may provide lower risks of postoperative myocardial infarction, pulmonary complications, gastric ileus, and mortality than general anesthesia [17-19]. 
Each patient's surgical and anesthetic plans must be carefully evaluated to optimize the operating room experience [20,21]. Patients undergoing surgical debridement in prone position require an extra level of attention to ensure airway patency, oxygenation, and ventilation throughout surgery (Figures 1, 2 and 3). Since sedation can lead to central and obstructive apneas, the jaw lift maneuver as seen in Figure 3 is one method to maintain airway patency. Anesthetic medications must be carefully selected to achieve therapeutic goals while avoiding respiratory depression and excessive muscle relaxation. The patient's medical history, such as whether he/she is paraplegic or suffering from incomplete neuropathy, will also determine the degree of antinociception and areflexia required at the surgical site. We also follow a specific protocol for timed skin checks in order to minimize skin injury from medical devices during procedures in the operating room.

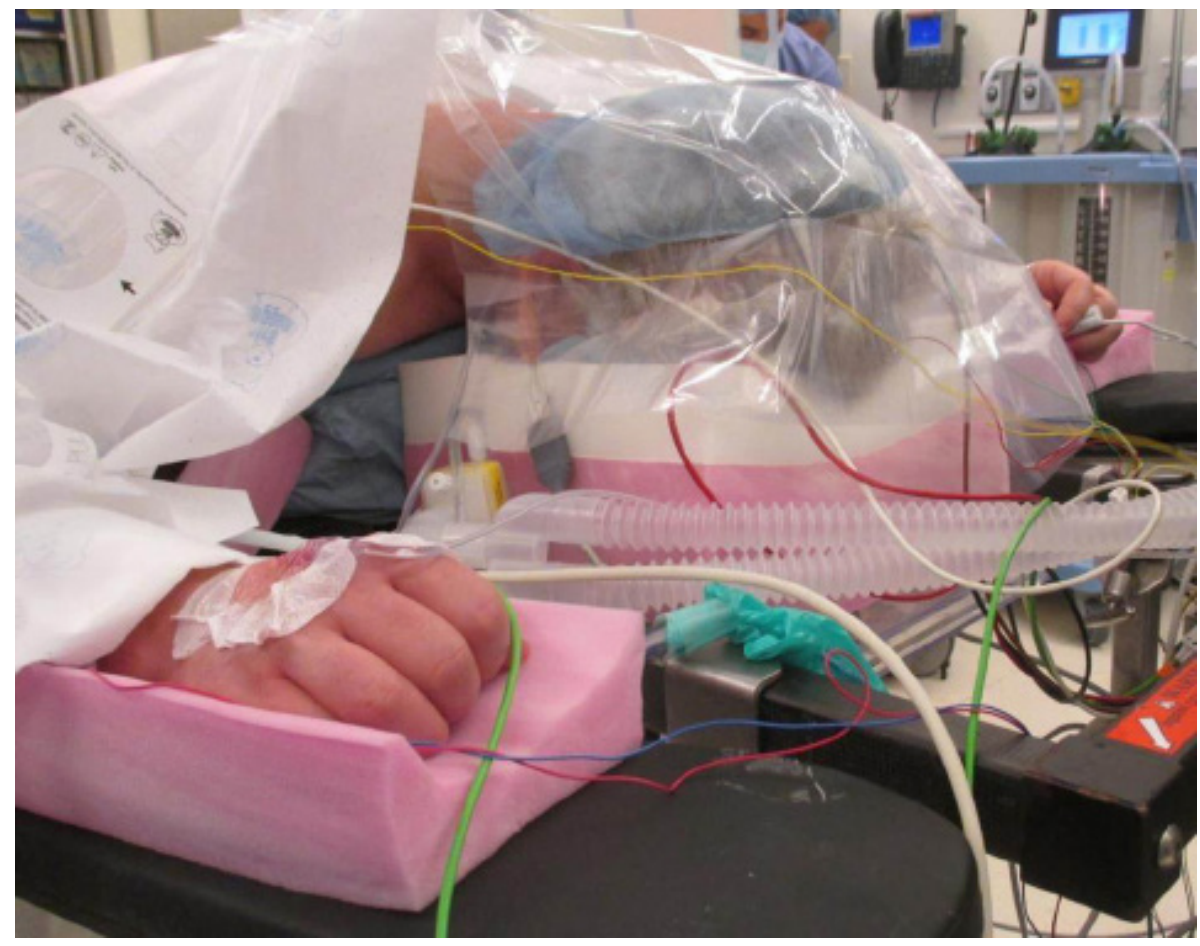

Figure 1: General Endotracheal Anesthesia in Prone Position Typically Used for Spine Surgery

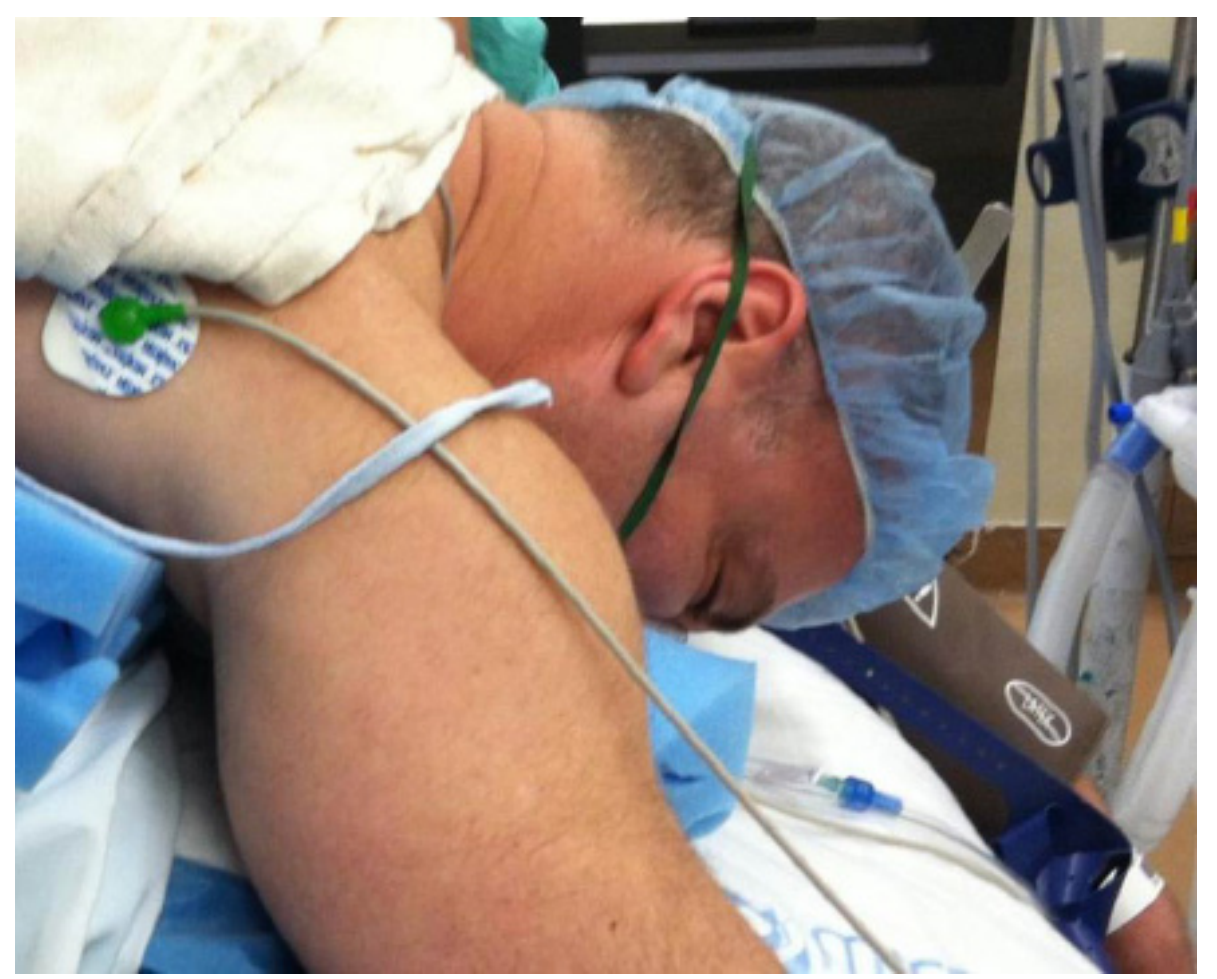

Figure 2: Face Mask Oxygenation and Capnography for Regional Anesthesia With Sedation in Prone PositionNote risk of mouth and nose occlusion from foam pad as well as tongue obstruction of pharynx 


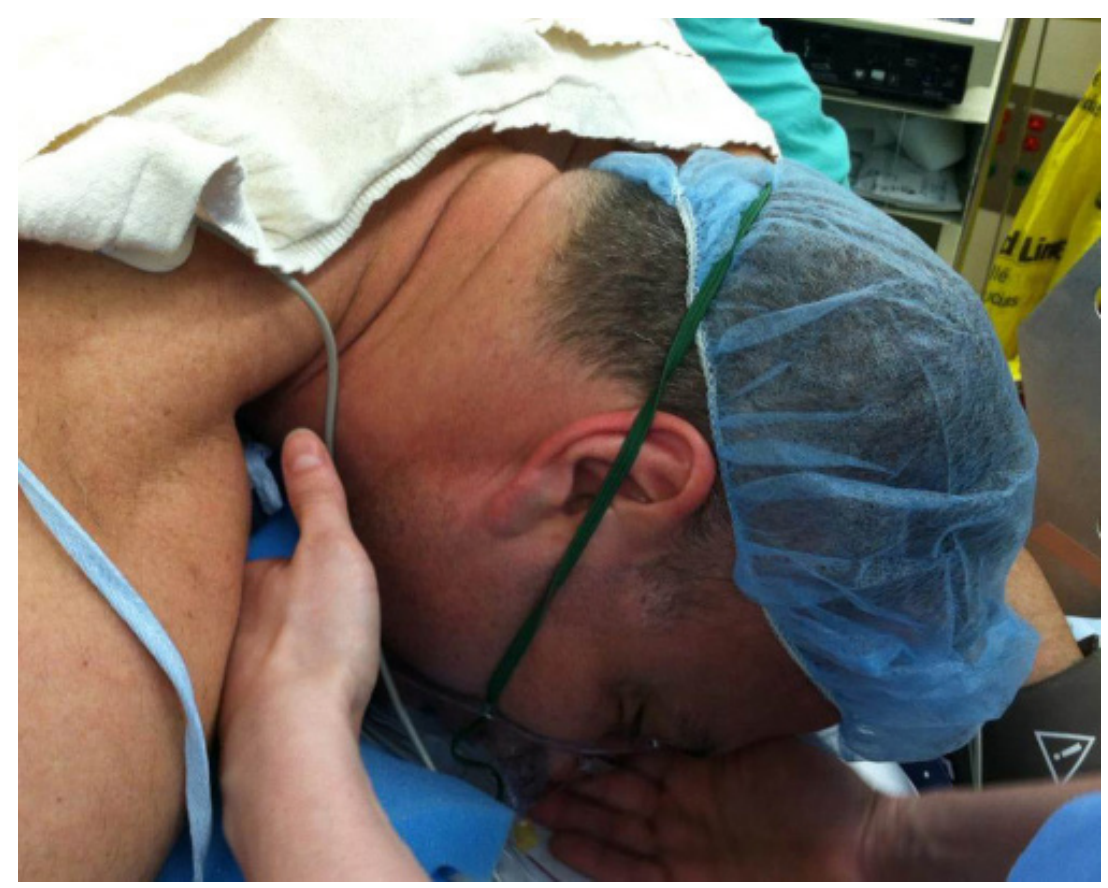

Figure 3: Jaw Lift in Prone Position

\section{Study Purpose}

We hypothesized that airway management for many patients undergoing surgical debridement in prone position can be accomplished by regional anesthesia without intubation, or if necessary, general endotracheal anesthesia without NMB when conditions are favorable. The purpose of our study was to review cases of patients undergoing sacral pressure ulcer debridement in prone position and to formulate an algorithm to determine whether regional anesthesia with sedation as needed is a reasonable alternative to general endotracheal anesthesia.

\section{Materials and Methods}

The study was performed with approval from the New York University School of Medicine Institutional Review Board. It is a retrospective chart review of the Wound Electronic Medical Record (WEMR) at New York University Langone Medical Center, an 806-bed teaching center located in a diverse urban setting and caring for a population with a broad spectrum of medical conditions. The chart review of 745 patients from July 2008 to January 2011 revealed several patient attributes which were essential for choosing an anesthesia technique. Therefore, we selected four diverse cases based on each individual's status that would allow us to demonstrate the wide-range of anesthetic techniques available to patients undergoing sacral pressure ulcer debridement in prone position. We then formulated an algorithm based on our selected cases and a review of the literature. The case review consisted of evaluating the indication and plan of the surgery, the patient's health status, the anesthetic method used, and any significant anesthesia-related events. The literature review focused on the benefits of regional over general anesthesia in patients with pressure ulcers, indications for various anesthetic techniques based on presenting circumstances, and the challenges of administering anesthesia in prone position. PubMed and Ovid databases were used for the literature search. Searched terms included combination of "prone position", "pressure ulcer", "prone anesthesia”, "prone airway", "surgical debridement”, “wound anesthesia", "regional anesthesia", "neuromuscular blocker". We reviewed approximately sixty articles for our study and mainly focused on randomized controlled trials, retrospective cohort studies, case control studies, case series, and case reports.

\section{Results}

We present four cases which required unique anesthesia management to successfully debride sacral pressure ulcers in prone position. No complications were observed in any of the cases. Figure 4 represents our algorithm for administering anesthesia based on the surgical plan and the degree of antinociception required. Our cases were carefully selected to represent all three major branches illustrated in Figure 4 (Complete Neuropathy, Sodium Channel Blockade, General Anesthesia). Figure 5 represents the traditional trajectory for endotracheal anesthesia in prone position surgery. Each step of the trajectory lists the commonly used airway management device, pharmacologic agents, and ventilation strategy. Figure 6 represents our own proposed trajectory for endotracheal anesthesia in prone position surgery. The major difference in our proposal is the exclusion of NMB and the use of video laryngoscopy during intubation. Table 1 expands upon the airway management algorithm section in Figure 4 and lists commonly used anesthetic drug combinations and airway management devices in prone position surgery. Table 1 is separated into three classes depending on the type of airway management device used. The right column identifies an optimal ventilation method based on the administered drugs and the left column represents protocol codes for referral purposes throughout this study. 


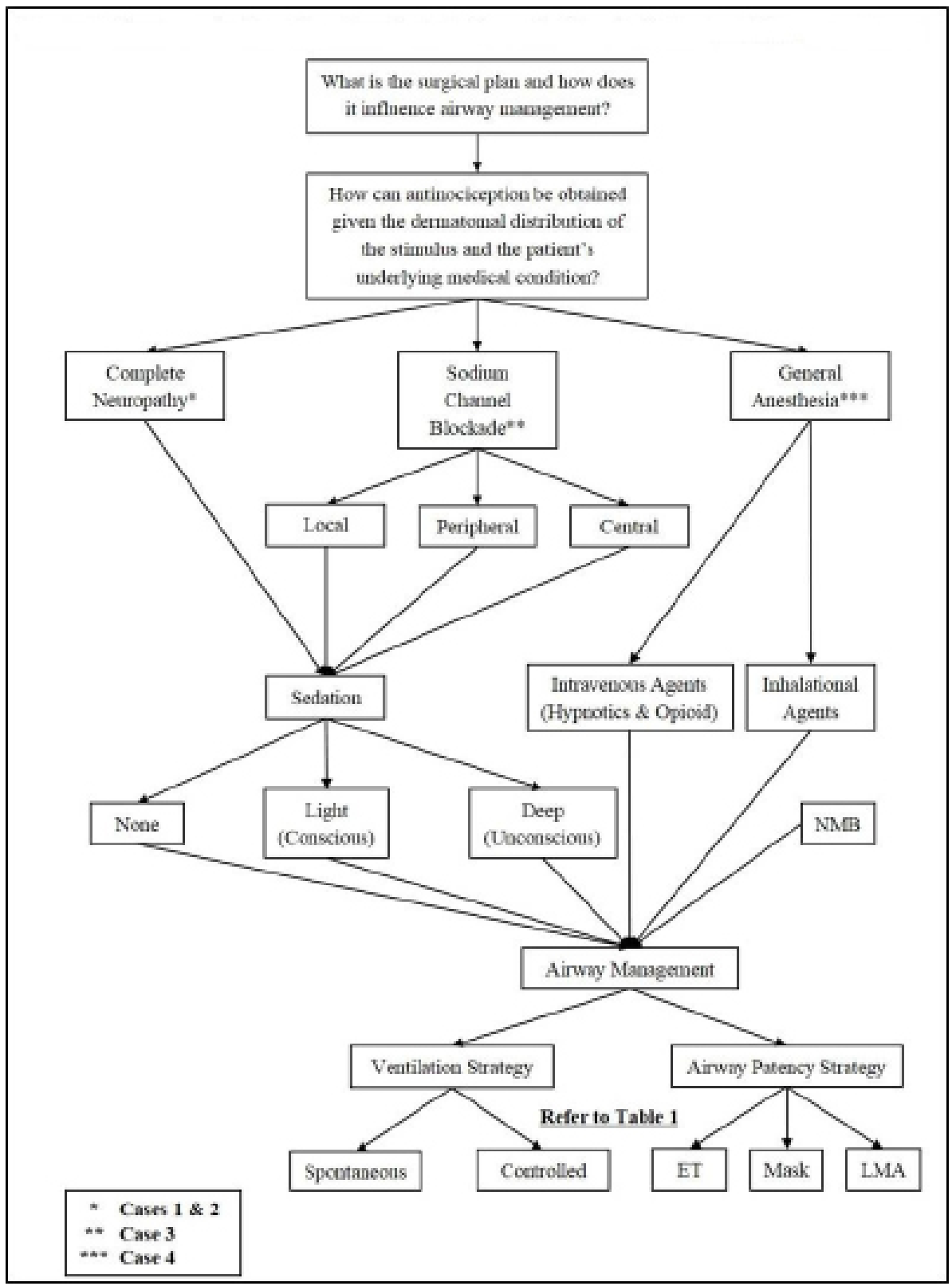

Figure 4: Algorithm for providing anesthesia in prone position for patients with pressure ulcers

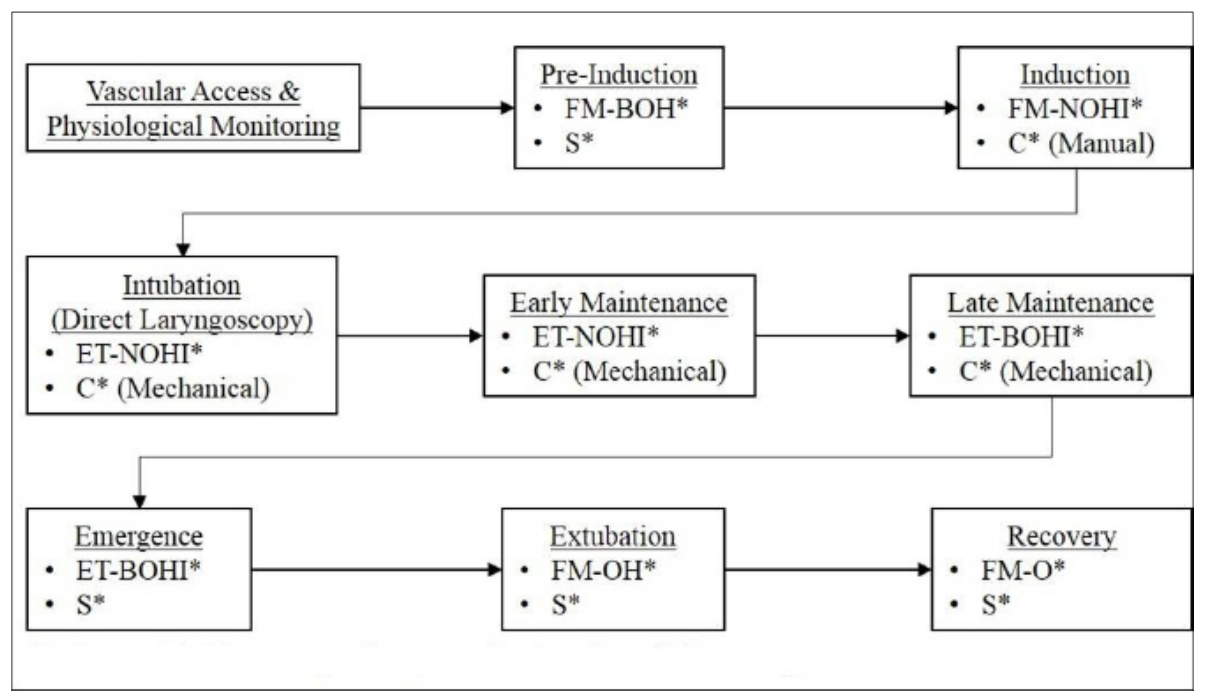

* Refer to Table 1 for corresponding protocol code and ventilation strategy

Figure 5: Traditional Trajectory for Endotracheal Anesthesia in Prone Position Surgery 


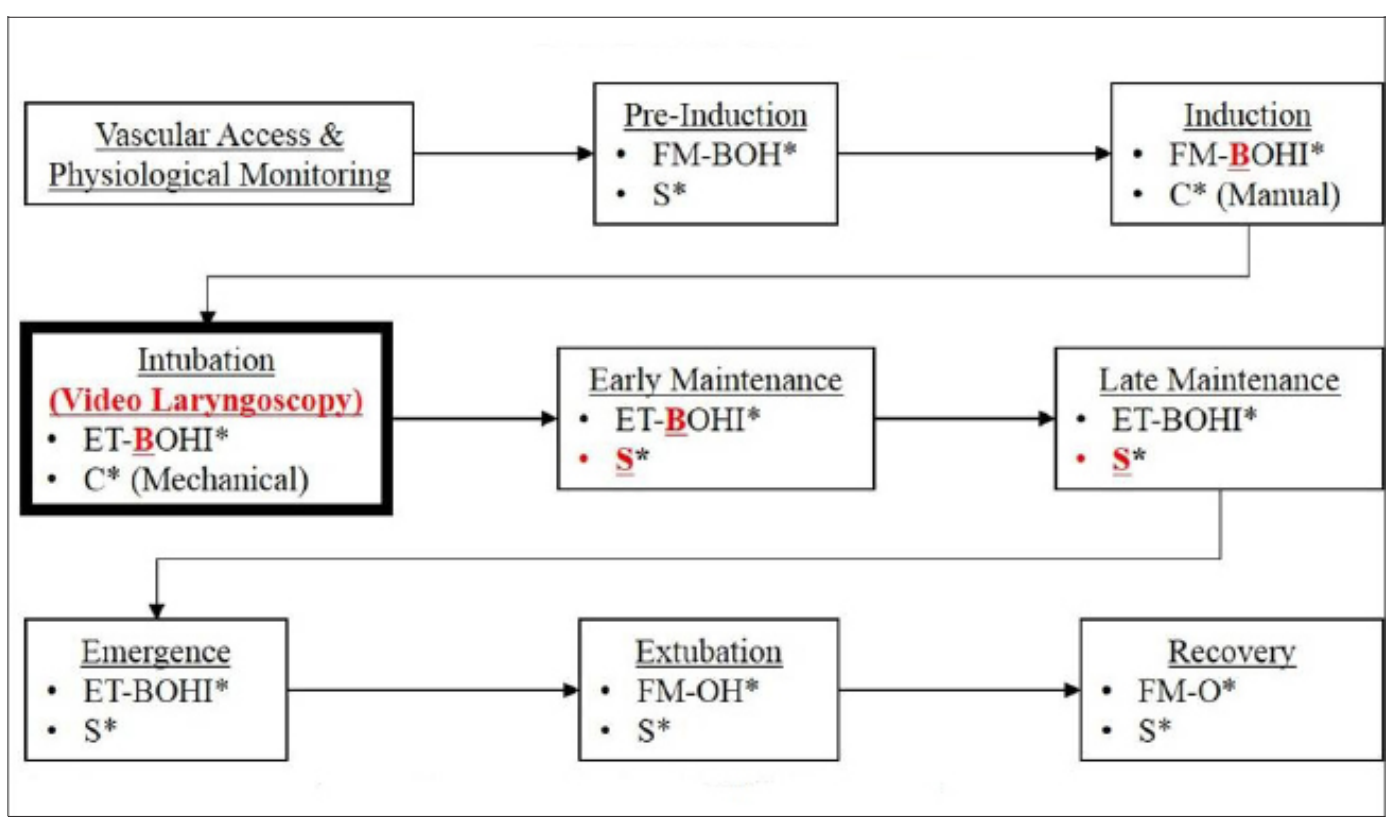

${ }^{*}$ Refer to Table 1 for corresponding protocol code and ventilation strategy

${ }^{*}$ Major changes compared to Figure are highlighted in red, bold, and underlined

Figure 6: Proposed Trajectory for Endotracheal Anesthesia in Prone Position Surgery

\begin{tabular}{|c|c|c|c|c|c|c|c|}
\hline Protocol Code & Airway Device & $\begin{array}{l}\text { NMB } \\
\text { Succinylcholine }^{1} \\
\text { Rocuronium }^{2} \\
\text { Vecuronium }^{2}\end{array}$ & $\begin{array}{c}\text { SCB } \\
\text { Lidocaine } \\
\text { Bupivacaine } \\
\text { Mepivacaine }\end{array}$ & $\begin{array}{c}\text { Opioid } \\
\text { Fentanyl } \\
\text { Remifentanil } \\
\text { Morphine }\end{array}$ & $\begin{array}{l}\text { Hypnotic } \\
\text { Propofol Etomidate } \\
\text { Ketamine }\end{array}$ & $\begin{array}{l}\text { Inhalational } \\
\text { Sevoflurane } \\
\text { Desflurane } \\
\text { Isoflurane }\end{array}$ & Ventilation \\
\hline FM- $\Phi$ & \multirow{7}{*}{ FM } & $x$ & $x$ & $x$ & $x$ & $x$ & S \\
\hline FM-BOH & & $x$ & $\sqrt{ }$ & $\sqrt{ }$ & $\sqrt{ }$ & $x$ & S \\
\hline FM-BOI & & $x$ & $\checkmark$ & $\checkmark$ & $x$ & $\checkmark$ & $\mathrm{S}$ \\
\hline FM-BOHI & & $x$ & $\checkmark$ & $\checkmark$ & $\checkmark$ & $\checkmark$ & S \\
\hline FM-NOH & & $\checkmark$ & $x$ & $\checkmark$ & $\checkmark$ & $x$ & $\mathrm{C}$ \\
\hline FM-NOI & & $\checkmark$ & $x$ & $\checkmark$ & $x$ & $\sqrt{ }$ & $\mathrm{C}$ \\
\hline FM-NOHI & & $\checkmark$ & $x$ & $\checkmark$ & $\checkmark$ & $\checkmark$ & $\mathrm{C}$ \\
\hline LMA-BOH & \multirow{6}{*}{ LMA } & $x$ & $\checkmark$ & $\checkmark$ & $\checkmark$ & $x$ & S \\
\hline LMA-BOI & & $x$ & $\checkmark$ & $\checkmark$ & $x$ & $\checkmark$ & S \\
\hline LMA-BOHI & & $x$ & $\checkmark$ & $\checkmark$ & $\checkmark$ & $\checkmark$ & S \\
\hline LMA-NOH & & $\checkmark$ & $x$ & $\checkmark$ & $\checkmark$ & $x$ & $\mathrm{C}$ \\
\hline LMA-NOI & & $\checkmark$ & $x$ & $\checkmark$ & $x$ & $\checkmark$ & $\mathrm{C}$ \\
\hline LMA-NOHI & & $\checkmark$ & $x$ & $\checkmark$ & $\checkmark$ & $\checkmark$ & $\mathrm{C}$ \\
\hline ET-BOH & \multirow{6}{*}{ ET } & $x$ & $\checkmark$ & $\checkmark$ & $\checkmark$ & $x$ & $\mathrm{~S}$ or $\mathrm{C}$ \\
\hline ET-BOI & & $x$ & $\checkmark$ & $\checkmark$ & $x$ & $\checkmark$ & $\mathrm{S}$ or $\mathrm{C}$ \\
\hline ET-BOHI & & $x$ & $\checkmark$ & $\checkmark$ & $\checkmark$ & $\checkmark$ & $\mathrm{S}$ or $\mathrm{C}$ \\
\hline ET-NOH & & $\checkmark$ & $x$ & $\checkmark$ & $\checkmark$ & $x$ & $\mathrm{C}$ \\
\hline ET-NOI & & $\checkmark$ & $x$ & $\checkmark$ & $x$ & $\checkmark$ & $\mathrm{C}$ \\
\hline ET-NOHI & & $\checkmark$ & $x$ & $\checkmark$ & $\checkmark$ & $\checkmark$ & $\mathrm{C}$ \\
\hline
\end{tabular}

- $\quad$ FM: Face Mask, LMA: Laryngeal Mask Airway, ET: Endotracheal Tube, N: Neuromuscular Blocker, B: Sodium Channel Blocker, O: Opioid, H: Hypnotic, I: Inhalational Agent, S: Spontaneous (negative pressure) Ventilation, C: Controlled (positive pressure) Ventilation, $\Phi$ : No Anesthetic Drugs Administered

- $\quad{ }^{1}$ Depolarizing NMB, ${ }^{2}$ Non-Depolarizing NMB

Table 1: Commonly Used Anesthetic Drug Combinations and Airway Management Devices in Prone Position Surgery 


\section{Case Discussions}

\section{Case 1}

Patient RP (complete neuropathy of spinal cord), Figure 7: The patient, a 55 year old female with history of spinal cord tumor excision, was completely insensate below the lesion at the thoracic level. She received general endotracheal anesthesia (ET-NOHI in Table 1) for the first wound debridement, mainly because of the anesthesiologist's limited understanding of the neuropathy. For subsequent debridement procedures, the patient refused sedation and positioned herself on the operating room table. No anesthetic agents were used (FM- $\Phi$ in Table 1). Wound was managed with secondary intention, offloading, and local wound care, as patient refused flap closure.

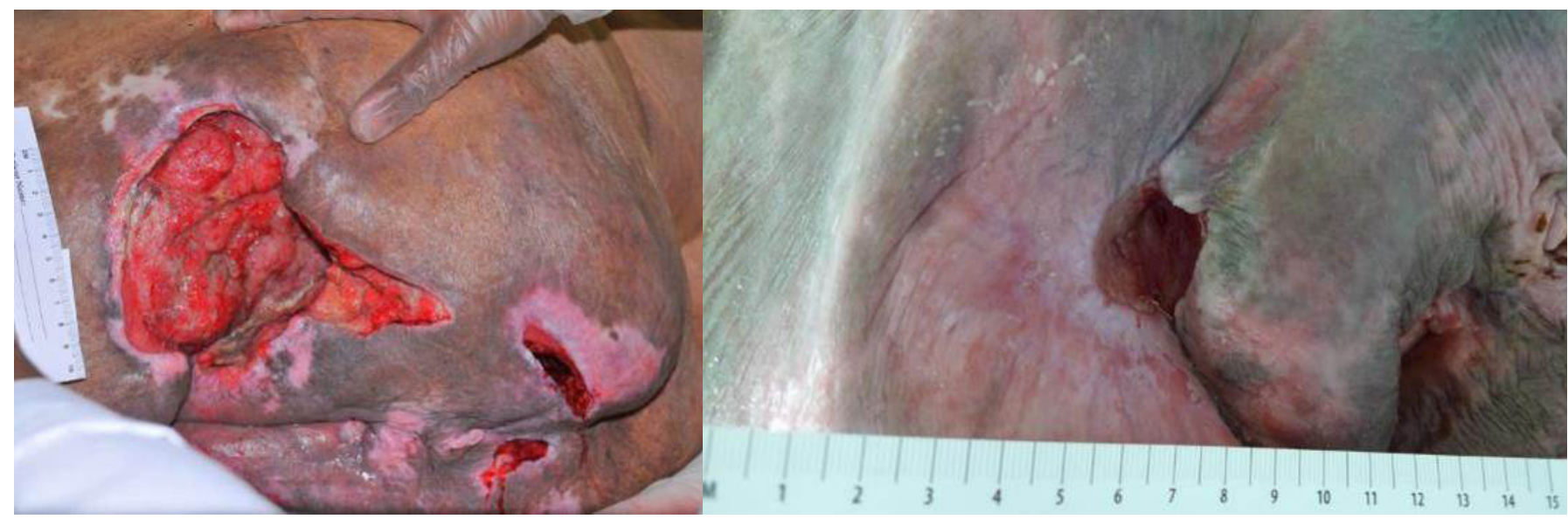

55 year old female with T10 spinal cord tumor excision, paraplegia, diabetes mellitus, obesity, hypothyroidism, right leg open reduction and internal fixation for fracture after a fall with non-healing wounds Ischium: $9.0 \mathrm{~cm}^{2}, 4.8 \mathrm{~cm}^{2}$

Sacrococcygeal: $16.0 \mathrm{~cm}^{2}$

Figure 7: Case 1, Patient RP

\section{Case 2}

Patient SC (complete neuropathy of spinal cord), Figure 8: The patient, a 44 year old male and a previously healthy smoker, suffered a fall at a construction site and was completely insensate due to traumatic paraplegia at the lumbar level. Although his complete neuropathy provided antinociception, the patient requested sedation (FM-BOH in Table 1) in order to be oblivious to the sounds of surgery and the odor of the cautery. Wound was managed with secondary intention, offloading, and local wound care, as patient refused flap closure.

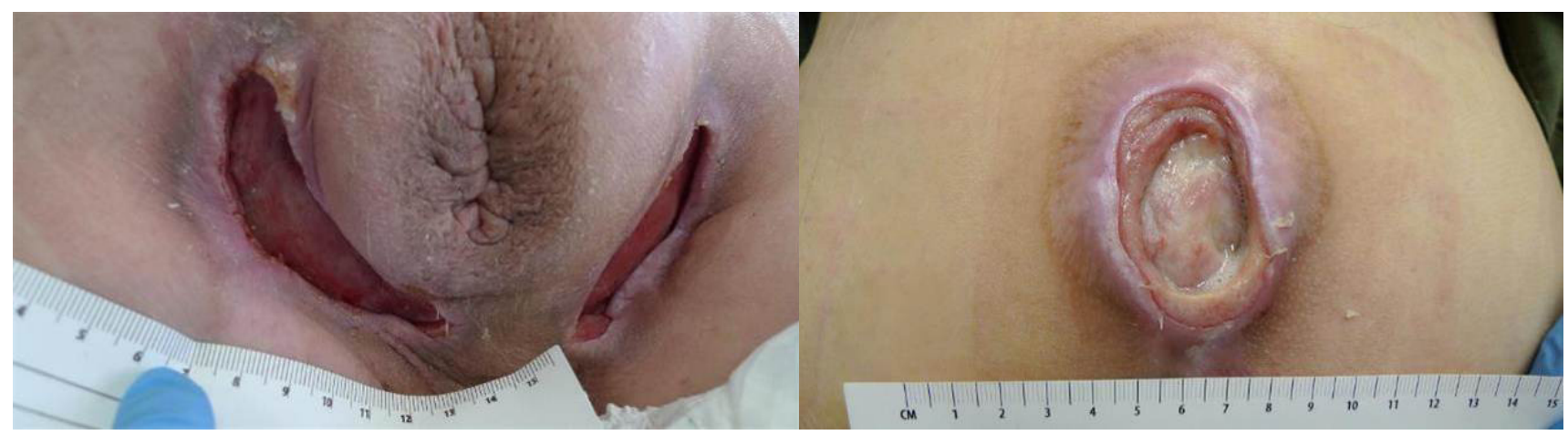

44 year old male construction worker with tobacco use, presenting after a fall resulting in paraplegia and non-healing wounds Ischium: $16.6 \mathrm{~cm}^{2}, 17.0 \mathrm{~cm}^{2}$

Sacrococcygeal: $2.1 \mathrm{~cm}^{2}, 1.0 \mathrm{~cm}^{2}$

Figure 8: Case 2, Patient SC

\section{Case 3}

Patient JG (incomplete neuropathy of spinal cord), Figure 9: The patient, a 53 year old male with no significant medical history, suffered a gunshot wound to his spinal cord at the lumbar level resulting in incomplete paraplegia. He required spinal anesthesia (FM-BOH in Table 1) to prevent autonomic hyperreflexia as well as intravenous sedation to minimize anxiety. Patient was lost to follow-up after the operation so wound could not be further evaluated. 


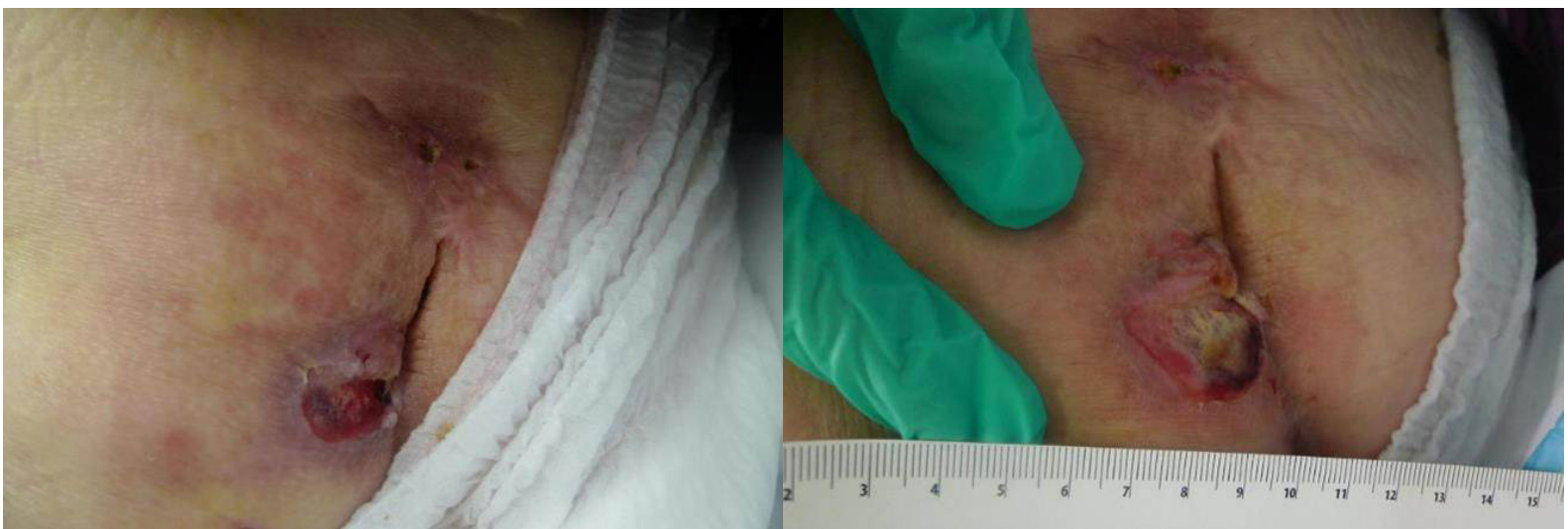

51 year old male with diabetes mellitus, tobacco use, paraplegia and non-healing wounds after a gunshot wound to the L4-5 region, status post flap to left ischium

Ischium: $7.8 \mathrm{~cm}^{2}, 2.6 \mathrm{~cm}^{2}$

Fifth toe, left: $1.6 \mathrm{~cm}^{2}$

Heel, left: $24.7 \mathrm{~cm}^{2}$

Leg, left: $1.7 \mathrm{~cm}^{2}, 3.1 \mathrm{~cm}^{2}, 2.4 \mathrm{~cm}^{2}$

Ankle, left: $2.8 \mathrm{~cm}^{2}, 1.3 \mathrm{~cm}^{2}, 6.1 \mathrm{~cm}^{2}$

Figure 9: Case 3, Patient JG

\section{Case 4}

Patient OS (incomplete neuropathy of spinal cord), Figure 10: The patient, a 39 year old male with no significant medical history, suffered a thoracolumbar spinal cord injury which was complicated by severe osteomyelitis from multi-drug resistant bacteria. As this was expected to be a prolonged procedure, he underwent a glidescope intubation following lidocaine topicalization of the airway, propofol sedation, spontaneous ventilation with synchronized intermittent mandatory ventilation (SIMV), and bispectral index (BIS) monitoring without the use of NMB (ET-BOH in Table 1). Wound was managed with secondary intention, offloading, and local wound care, as patient was not a candidate for flap closure due to insufficient tissue availability.

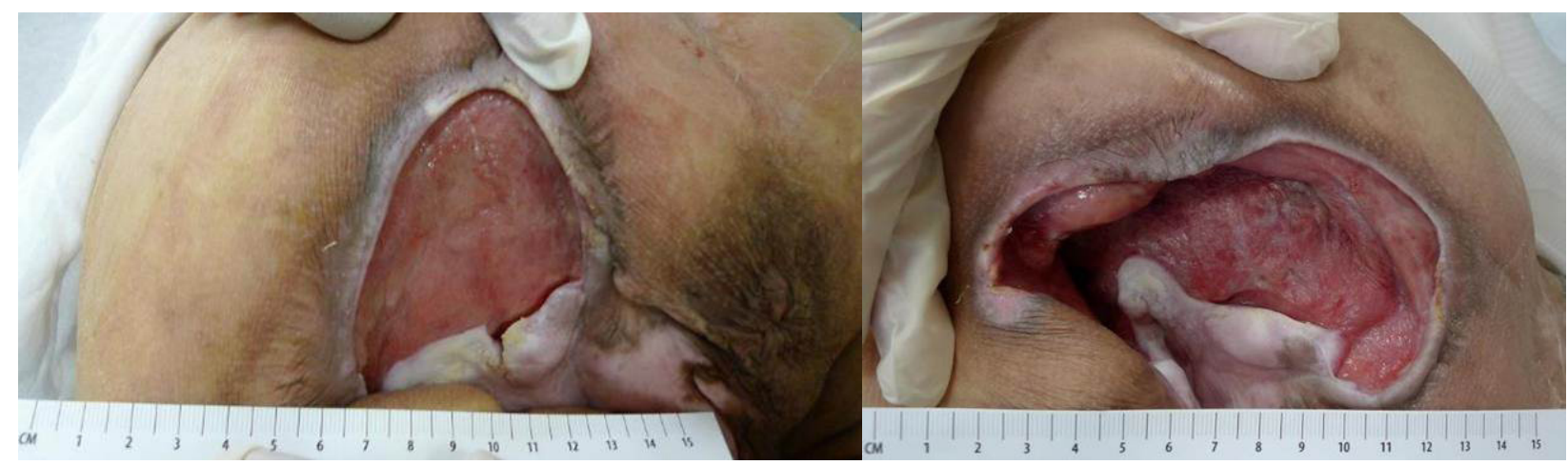

39 year old male with paraplegia after a gunshot wound and history of two failed gluteal flap procedures Ischium: $28.8 \mathrm{~cm}^{2}, 16.5 \mathrm{~cm}^{2}$

Figure 10: Case 4, Patient OS

\section{Discussion}

\section{Patient Attributes \& Anesthesia Decisions}

Patients with chronic pressure ulcers require prompt diagnosis and treatment in order to avoid further complications. Surgical debridement is a crucial component of wound management as it removes devitalized and contaminated tissues that may ultimately inhibit immune defenses and encourage infection [22].

Intraoperative anesthesia for pressure ulcer debridement in the prone position presents unique challenges, including deciding whether general endotracheal anesthesia with NMB is necessary at all. Depending on the technique chosen, this position may present a variety of procedural and physiologic issues, such as difficult mask fit, decreased cardiac index, increased blood loss, and reduction of pulmonary compliance [11,13]. Our review demonstrates the variability of pharmacologic and airway management requirements in these patients. Cases 1 to 3 were performed successfully without intubation, while case 4 required intubation due to the expected prolonged procedure time and the risk of significant blood loss. Based on our research and the algorithm in Figure 4, our airway management consisted of avoiding endotracheal intubation, excluding all protocol codes beginning with ET- (Table 1), whenever possible on the premise that complications associated with intubation are worth minimizing. 
Cases 1 and 2 represent patients who were completely insensate due to paraplegia. Patients with spinal cord injury always need individualized assessment of their anesthetic requirement depending on the severity of their impairment. As these two cases involved complete neuropathy, it was determined that providing antinociception and areflexia by pharmacologic agents was unnecessary and only monitored anesthesia care would be performed. This was in accordance with a review article which suggested that patients with loss of sensation caused by spinal cord injury could undergo surgery below the level of injury without anesthesia and without feeling pain from the operative site [23]. Despite this, some prefer to be sedated due to fear of mutilation and aversion to the smell of burning tissues, as seen in case 2, while some prefer to be alert and awake, as seen in case 1.

Case 3 involved a patient with incomplete paraplegia from a spinal cord injury. In the absence of complete neuropathy, antinociception and areflexia can be achieved with regional anesthesia using a sodium channel blocker (SCB), also referred to as a "local anesthetic" in various textbooks. Regional anesthesia can be administered either locally, peripherally, or centrally (Figure 4). Unlike NMBs which bind to efferent neurons to produce paralysis, SCB mainly binds to voltage-gated sodium channels on afferent neurons to produce a temporary interruption of nerve function, inducing antinociception and areflexia. A major benefit of SCB is its analgesic effect from spinal deafferentation, which subsequently decreases anesthetic requirement for sedation [24]. Several studies have demonstrated that their use can lower the amount of inhalational and hypnotic agents needed to achieve adequate depth of anesthesia $[25,26]$. As incomplete spinal cord injury has a risk of somatic pain in the absence of regional block, we believe SCB is valuable in providing optimal anesthesia.

\section{Autonomic Dysreflexia}

Another concern for patients with spinal cord injury is autonomic dysreflexia (AD). Frequently observed in patients with injury above the T6 level as a result of exaggerated sympathetic excitation, AD is a clinical emergency with possible fatal complications [27]. The spinal cord ends at vertebral levels T12-L1 in adults. A common episode is characterized by acute hypertension, cardiac dysrhythmia, headache, convulsions, and rarely, intracranial hemorrhage [28]. In addition to providing antinociception and areflexia for our patient in case 3, it was imperative to recognize that surgical debridement could possibly initiate an episode of AD. Two observational studies have found that up to $90 \%$ of patients with spinal cord injury undergoing surgery with topical anesthesia or no anesthesia can develop $\mathrm{AD}$, but this incidence can be significantly reduced by general or spinal anesthesia [29]. Injuries at the lumbar level are not associated with autonomic dysreflexia, since they involve peripheral nerves in the cauda equina. Our patient successfully underwent the debridement with spinal anesthesia and intravenous sedation for anxiety without airway compromise (FM-BOH in Table 1).

\section{Endotracheal Intubation}

Case 4 represents a scenario where endotracheal intubation was necessary to secure a patent airway, considering the duration of the surgery and the risk of blood loss from a severe infection. Traditionally in such patients, endotracheal anesthesia is carried out in a similar manner to the trajectory in Figure 5, in which NMB is administered during induction to aid in intubation by direct laryngoscopy. However, we believe NMB should be avoided in endotracheal anesthesia unless clinically indicated. The ASA's difficult airway management guidelines emphasize the importance of maintaining spontaneous ventilation until the airway is secured with endotracheal intubation by avoiding NMB, thereby reducing the risks of apnea and areflexia [30]. Although this technique is usually reserved for patients categorized as "difficult airway" or "difficult direct laryngoscopy", it can also be applied to patients with "easy airway" or "low-risk direct laryngoscopy".

Studies have shown that propofol in combination with fentanyl, alfentanil, or remifentanil, can decrease pharyngeal and laryngeal reactivity to provide adequate conditions for intubation without the potential adverse effects of NMB [31,32]. Although shortacting NMB such as succinylcholine, a depolarizing muscle relaxant, can facilitate intubation and reduce complications associated with airway management, it may be associated with undesirable sequelae including hyperkalemia, especially in spinal cord injured patients; hypersensitivity, cardiac arrhythmias, and malignant hyperthermia [33,34]. Patients with pressure ulcers are especially vulnerable to these adverse effects as they are commonly debilitated with a high risk of developing sarcopenia. Sarcopenia, along with various pathologic states, causes up-regulation of acetylcholine receptors throughout the muscle membrane, leading to higher potassium efflux and lethal hyperkalemia when succinylcholine is administered [35,36]. Therefore, we advise avoiding succinylcholine in the majority of patients with pressure ulcers. Non-depolarizing muscle relaxants such as rocuronium and vecuronium are also associated with residual neuromuscular weakness due to their prolonged half-life of 60-95 minutes, which can be significantly extended if a patient has liver or renal impairment [10,37]. These agents can often outlast a brief surgical wound debridement and lead to respiratory failure and reintubation during post-anesthesia care.

Our case 4 patient successfully received endotracheal anesthesia without NMB following the protocol ET-BOH (Table 1). Two studies observing somatic and sympathetic responses to surgical incision found that administration of fentanyl lowered the levels of sevoflurane and propofol required to eliminate patient movement and hemodynamic changes without NMB [38,39]. Based on these findings and our selected cases, we propose that patients in favorable conditions undergoing surgical debridement with endotracheal anesthesia should follow the trajectory in Figure 6 as well as protocol codes ET-BOH, ET-BOI, or ET-BOHI (Table 1) during intubation. 


\section{Our Proposed Algorithm}

The major difference in our proposed trajectory in Figure 6 as compared to Figure 5 is the exclusion of NMB and the use of video laryngoscopy during intubation. As muscle paralysis is not used to assist intubation, we recommend using video laryngoscopy as it provides enhanced glottic visualization, a higher success rate, faster intubation time, and less need for cervical spine mobility than standard laryngoscopes [40,41]. Our suggested protocol codes, ET-BOH, ET-BOI, and ET-BOHI (Table 1) provide maximum airway control by the endotracheal tube, while benefitting from the pharmacologic combination of hypnotic, opioid, and inhalational agents without NMB. Commonly used with face mask and laryngeal mask airway (LMA), this combination of drugs offers the choice of ventilation strategy without the obligation to use controlled (positive-pressure) ventilation, as seen with protocol codes ET-NOH, ET-NOI, ET-NOHI (Table 1) [42]. If controlled ventilation is needed to prevent significant hypoventilation due to unfavorable resistance or compliance factors, patient sensing trigger mode such as SIMV should be used to prevent patient-ventilator asynchrony. Transitioning from SIMV to unsupported spontaneous ventilation during emergence can provide an opportunity for both opioid titration as well as assessment of pre-extubation pulmonary criteria. This allows the anesthesiologist to minimize the amount of anesthetic drugs administered and their adverse effects, all the while precisely timing the extubation for the intended goal of minimizing the duration of post-procedural mechanical ventilation [43]. This approach can provide safe and effective anesthesia even in severely ill patients.

Overall, our retrospective chart review provides valuable information in approaching patients with specific surgical indications. However, further studies are required to enhance our knowledge and care in patients with pressure ulcers given its wide range of possible clinical presentation. A randomized, stratified prospective clinical trial focusing on providing regional anesthesia with sedation as needed is recommended for further validation.

\section{Conclusion}

In summary, surgical debridement may be an essential component in the healing of sacral pressure ulcers under the WBP model. As the prone surgical position carries numerous challenges, developing a tailored anesthetic plan based on individual patient's comorbidities, severity of the wound, and general health status is critical in achieving a successful outcome. We believe patients in favorable conditions undergoing surgical debridement in prone position can be safely and successfully managed with monitored anesthesia care, regional anesthesia with sedation as needed, or if necessary, general endotracheal anesthesia without NMB. This especially holds true if complete neuropathy or SCB can provide adequate antinociception and areflexia without unnecessary pharmacologic intervention. The prone surgical position can also be problematic because of the risks of airway device related skin and mucosal injuries. As limited literature exists emphasizing this anesthesia technique for surgical debridement in prone position, we hope our work will contribute to providing quality anesthetic care for patients with pressure ulcers.

\section{Acknowledgement}

The authors would like to acknowledge the support of the National Institutes of Health (NIH) for the Diabetic and Pressure Ulcer Databank (R01 LM008443 04), Harold Brem, MD., and Thomas J.J. Blanck, M.D., Ph.D..

\section{References}

1. VanGilder C, Amlung S, Harrison P, Meyer S (2009) Results of the 2008-2009 International Pressure Ulcer Prevalence Survey and a 3-year, acute care, unitspecific analysis. Ostomy Wound Manage 1: 39-45.

2. Horn SD, Bender SA, Bergstrom N, Cook AS, Ferguson ML, et al. (2004) Description of the National Pressure Ulcer Long-Term Care Study. J Am Geriatr Soc 50: $1816-25$

3. National Pressure Ulcer Advisory Panel (2012) Pressure ulcers: Prevalence, Incidence and Implications for the Future. Washington, DC: NPUAP.

4. Lyder CH, Ayello EA (2009) Annual checkup: the CMS pressure ulcer present-on-admission indicator. Adv Skin Wound Care 22: 476-84.

5. Wall BM, Mangold T, Huch KM, Corbett C, Cooke CR (2003) Bacteremia in the chronic spinal cord injury population: risk factors for mortality. J Spinal Cord Med 26: 248-53.

6. Duncan KD (2007) Preventing pressure ulcers: the goal is zero. Jt Comm J Qual Patient Saf 33: 605-10.

7. Schiffman J, Golinko MS, Yan A, Flattau A, Tomic-Canic M, et al. (2009) Operative debridement of pressure ulcers. World J Surg 33: 1396-402.

8. Schultz GS, Sibbald RG, Falanga V, Ayello EA, Dowsett C, et al. (2003) Wound bed preparation: a systematic approach to wound management. Wound Repair Regen 11: S1-28.

9. Sibbald RG, Goodman L, Woo KY, Krasner DL, Smart H, et al. (2011) Special considerations in wound bed preparation 2011: an update. Adv Skin Wound Care 24: 415-36.

10. O’Neill D, Robins B, Ayello E, Cuff G, Linton P, et al. (2012) Regional anaesthesia with sedation protocol to safely debride sacral pressure ulcers. Int Wound J 9: 525-43.

11. Edgcombe H, Carter K, Yarrow S (2008) Anaesthesia in the prone position. Br J Anaesth 100: 165-83.

12. O'Neill DK, Maggi J (2009) Anesthetic care for patients with skin breakdown. Anesthesiol Clin 27: 599-603.

13. Agrawal S, Sharma JP, Jindal P, Sharma UC, Rajan M (2007) Airway management in prone position with an intubating Laryngeal Mask Airway. J Clin Anesth 19: $293-5$.

14. Weksler N, Klein M, Rozentsveig V, Weksler D, Sidelnik C, et al. (2007) Laryngeal mask in prone position: pure exhibitionism or a valid technique. Minerva Anestesiol 73: 33-7. 
15. Wilson J, Collins AS, Rowan BO (2012) Residual Neuromuscular Blockade in Critical Care. Crit Care Nurse 32: e1-9.

16. Ostergaard D, Engbaek J, Viby-Mogensen J (1989) Adverse reactions and interactions of the neuromuscular blocking drugs. Med Toxicol Adverse Drug Exp 4: 351-68.

17. Rodgers A, Walker N, Schug S, McKee A, Kehlet H, et al. (2000) Reduction of postoperative mortality and morbidity with epidural or spinal anaesthesia: results from overview of randomised trials. BMJ 16: 1493.

18. Freise H, Fischer LG (2009) Intestinal effects of thoracic epidural anesthesia. Curr Opin Anaesthesiol 22: 644-8.

19. van Lier F, van der Geest PJ, Hoeks SE, van Gestel YR, Hol JW, et al. (2011) Epidural analgesia is associated with improved health outcomes of surgical patients with chronic obstructive pulmonary disease. Anesthesiology 115: 315-21.

20. Buchanan K, Bernstein N, Ayello EA, O’Neill DK (2011) Cardiac device interrogation for safer care of surgical wound patients. Adv Skin Wound Care 24: 507-14.

21. Yoo C, Ayello EA, Robins B, Salamanca VR, Bloom MJ, et al. (2014) Perioperative use of bispectral (BIS) monitor for a pressure ulcer patient with locked-in syndrome (LIS). Int Wound J 11: 540-5.

22. Cardinal M, Eisenbud DE, Armstrong DG, Zelen C, Driver V, et al. (2009) Serial surgical debridement: A retrospective study on clinical outcomes in chronic lower extremity wounds. Wound Repair Regen 17: 306-11.

23. Kanonidou Z (2006) Anaesthesia for chronic spinal cord lesions. Hippokratia 10: 28-31.

24. Gentili M, Huu PC, Enel D, Hollande J, Bonnet F (1998) Sedation depends on the level of sensory block induced by spinal anaesthesia. Br J Anaesth 81: 970-1. 25. Hodgson PS, Liu SS (2001) Epidural lidocaine decreases sevoflurane requirement for adequate depth of anesthesia as measured by the Bispectral Index monitor. Anesthesiology 94: 799-803.

26. Shariat-Moharari R, Samadi A, Imani F, PanahKhahi M, Khashayar P, et al. (2013) The Effect of Epidural Bupivacaine on BIS Levels in the Awake Phase and on the Maintenance Doses of Propofol and Fentanyl During General Anesthesia. Anesth Pain Med 2: 149-53.

27. Bycroft J, Shergll IS, Chung EA, Arya N, Shah PJ (2005) Autonomic dysreflexia: a medical emergency. Postgrad Med J 81: 232-5.

28. Krassioukov A, Warburton DE, Teasell R, Eng JJ, Spinal Cord Injury Rehabilitation Evidence Research Team (2009) A systematic review of the management of autonomic dysreflexia after spinal cord injury. Arch Phys Med Rehabil 90: 682-95.

29. Eltorai IM, Wong DH, Lacerna M, Comarr AE, Montroy R (1997) Surgical aspects of autonomic dysreflexia. J Spinal Cord Med 20: 361-4.

30. Apfelbaum JL, Hagberg CA, Caplan RA, American Society of Anesthesiologists Task Force on Management of the Difficult Airway (2013) Practice guidelines for management of the difficult airway: an updated report by the American Society of Anesthesiologists Task Force on Management of the Difficult Airway. Anesthesiology 118: 251-70.

31. Bonnin M, Therre P, Albuisson E, Beaujard H, Barthelemy I, et al. (2007) Comparison of a propofol target-controlled infusion and inhalational sevoflurane for fibreoptic intubation under spontaneous ventilation. Acta Anaesthesiol Scand 51: 54-9.

32. Taha S, Siddik-Sayyid S, Alameddine M, Wakim C, Dahabra C, et al. (2005) Propofol is superior to thiopental for intubation without muscle relaxants. Can J Anaesth 52: 249-53.

33. Zhang B, Hepner DL, Tran MH, Friedman M, Korn JR, et al. (2009) Neuromuscular blockade, reversal agent use, and operating room time: retrospective analysis of US inpatient surgeries. Curr Med Res Opin 25: 943-50.

34. Claudius C, Garvey LH, Viby-Mogensen J (2009) The undesirable effects of neuromuscular blocking drugs. Anaesthesia 64: 10-21.

35. Martyn JA, Richtsfeld M (2009) Succinylcholine-induced Hyperkalemia in Acquired Pathologic States: Etiologic Factors and Molecular Mechanisms. Anesthesiology 104: 158-69.

36. Matthews JM (2000) Succinylcholine-induced hyperkalemia and rhabdomyolysis in a patient with necrotizing pancreatitis. Anesth Analg 91: 1552-4.

37. Tripathi SS, Hunter JM (2006) Neuromuscular blocking drugs in the critically ill. Contin Educ Anaesth Crit Care Pain 6: 119-23.

38. Katoh T, Kobayashi S, Suzuki A, Iwamoto T, Bito H, et al. (1999) The effect of fentanyl on sevoflurane requirements for somatic and sympathetic responses to surgical incision. Anesthesiology 90: 398-405.

39. Smith C, McEwan AI, Jhaveri R, Wilkinson M, Goodman D, et al. (1994) The interaction of fentanyl on the Cp50 of propofol for loss of consciousness and skin incision. Anesthesiology 81: 820-8.

40. Jungbauer A, Schumann M, Brunkhorst V, Borgers A, Groeben H (2009) Expected difficult tracheal intubation: a prospective comparison of direct laryngoscopy and video laryngoscopy in 200 patients. Br J Anaesth 102: 546-50.

41. Griesdale DE, Liu D, McKinney J, Choi PT (2012) Glidescope ${ }^{\varpi}$ video-laryngoscopy versus direct laryngoscopy for endotracheal intubation: a systematic review and meta-analysis. Can J Anaesth 59: 41-52.

42. Ng A, Raitt DG, Smith G (2002) Induction of anesthesia and insertion of a laryngeal mask airway in the prone position for minor surgery. Anesth Analg 94: 1194-8.

43. Riem N, Boet S, Tritsch L, Bould D (2011) LMA with positive pressure ventilation is safe! Korean J Anesthesiol 61: 88-9. 


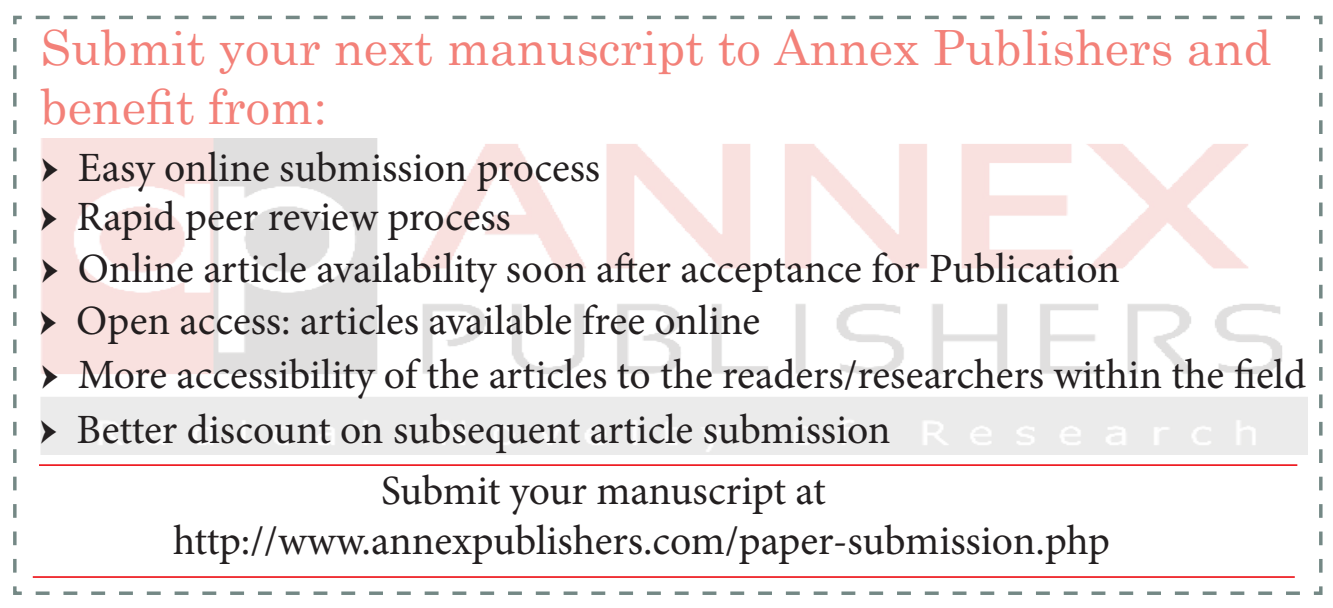

